\title{
S-bands of Jovian decametric emission
}

\author{
O. V. Arkhypov ${ }^{1}$ and H. O. Rucker ${ }^{2}$ \\ 1 Institute of Radio Astronomy, National Academy of Sciences of Ukraine, Chervonopraporna 4, 61002 Kharkiv, Ukraine \\ e-mail: rai@ri.kharkov.ua \\ 2 Space Research Institute, Austrian Academy of Sciences, Schmiedlstrasse 6, 8042 Graz, Austria \\ e-mail: rucker@oeaw.ac.at
}

Received 2 November 2007 / Accepted 1 February 2008

\section{ABSTRACT}

\begin{abstract}
There are theoretical and experimental arguments for a modulation of Jovian decametric S-emission by standing Alfvén waves in the regions of radio sources. One effect of such modulation is the concentration of S-burst emission around certain frequencies (Sbands) in dynamic spectra of radio emission. It is argued that S-bands are generated at the local electron cyclotron frequencies, around nodes of a standing Alfvén wave. To test this hypothesis, we derived several verifiable predictions: the anti-correlation of radio-flux in adjacent S-bands at short time scales $(<0.1 \mathrm{~s})$; the correlation of frequency shifts of S-bands at long time scales $(>0.1 \mathrm{~s})$; and the transformation of the broadband S-storm into S-bands. We searched for and found these effects experimentally. Because the predictions are confirmed, the standing wave modulation of Jovian S-emission appears valid.
\end{abstract}

Key words. planets and satellites: individual: Jupiter - magnetohydrodynamics (MHD) - plasmas - magnetic fields - waves radiation mechanisms: non-thermal

\section{Introduction}

Short (S) bursts of Jovian decametric emission (DAM) with the time scale of $\tau \sim 10^{-3} \mathrm{~s}$ form trains or bands near certain frequencies in dynamic spectra. Previous studies focused mainly on the microstructure of such trains with a time scale of $10^{-6}<\tau<10^{-1}$ s and a frequency band of $\Delta f<3 \mathrm{MHz}$ (see references in Litvinenko et al. 2004). However, previous studies did not focus on the trains' macrostructure $(\tau>0.1 \mathrm{~s}$ and/or $\Delta f>6 \mathrm{MHz}$ ), and obtained different interpretations.

Leblanc \& Genova (1981) found that the S-burst emission in dynamic spectra is arranged into a pattern of repetitive features, drifting to low frequencies with time. These features were interpreted as incomplete arcs with late vertex which reflect the radiation pattern in the form of thin conical sheets. Analogical arcs of long (L; $\tau \sim 1$ s) DAM bursts show certain frequency drift at $f>20 \mathrm{MHz}$ : positive in Io-B storms and negative in IoAC spectra (Leblanc 1981). However, the S-structures, drifting to high- or low-frequencies or without any drift, were observed in the Io-B storm of January 4, 1993 by three observatories simultaneously (Boudjada et al. 1995). Moreover, after averaging many $\mathrm{S}$-storms it was found that $\mathrm{S}$-emission concentrates near certain frequencies (Ryabov et al. 1985). Hence, the macrostructure of S-emission is more complicated than the arc pattern.

Some authors considered S-trains as a system that reflects wave disturbances of the radio-source's magnetic lines. Quasiperiodic bulbs moving along the Io-flux tube (Ryabov et al. 1997) or magnetohydrodynamic waves on its border (Boev et al. 1993) were suggested. The standing Alfvén wave in the Jovian ionospheric resonator is considered as a stimulus for S-burst generation (Ergun et al. 2006). In previous paper, we confirmed that average oscillations of S-emission probability with frequency corresponds to a standing Alfvén wave (Arkhypov \& Rucker 2006).
We test here new predictions of the standing Alfvén wave hypothesis on the micro-structural (Sect. 2) and macro-structural (Sect. 3) levels. The results are in favor of the connection of S-bands with nodes of standing modulation waves (Sect. 4).

\section{Microstructure}

According to the standard model (e.g., Zarka 1998), S-bursts are generated on the active magnetic lines, which are activated by the Io satellite (Fig. 1). The frequency $f$ of each component of the emitted band is approximately equal to the local electron cyclotron frequency $f_{\text {ce }}$ at the point of the active line from which it was emitted. Hence, the decametric sources are localized in the subpolar magnetosphere, at altitudes $\sim 0.1 R_{\mathrm{J}}$, where $R_{\mathrm{J}}=71372 \mathrm{~km}$ is Jupiter's equatorial radius. Io stimulates emission with some time delay. Accordingly, during this delay Io's orbital longitude increases on the lead angle $\Delta \lambda_{\text {Io }}$ in the frame of the Jovian magnetic field.

Usually, we found from 1 to $3 \mathrm{~S}$-trains or S-bands at different frequencies simultaneously in a dynamic spectrum of DAM (Fig. 2). If there are arc emissions from different active magnetic tubes at various longitudes, any correlation between radio fluxes in microstructures of adjacent S-trains is not expected.

However, the standing Alfvén wave can make quasiperiodical regions of maximal parallel electric currents and maximal transversal magnetic disturbances along one magnetic tube. Such regions coincide with wave nodes, where the transversal plasma motion is minimal, but the magnetic deflection from undisturbed direction is maximal. Sometimes we observe S-burst emission, mainly from these regions, as S-band illusions, if the Alfvén wave sufficiently alters the direction of narrow radio beams in the same regions (Fig. 3).

Such alteration is possible in terms of electron-cyclotron maser instability, which is considered the most promising explanation of S-bursts (Zarka 1998; Hess et al. 2007b). A resonant 


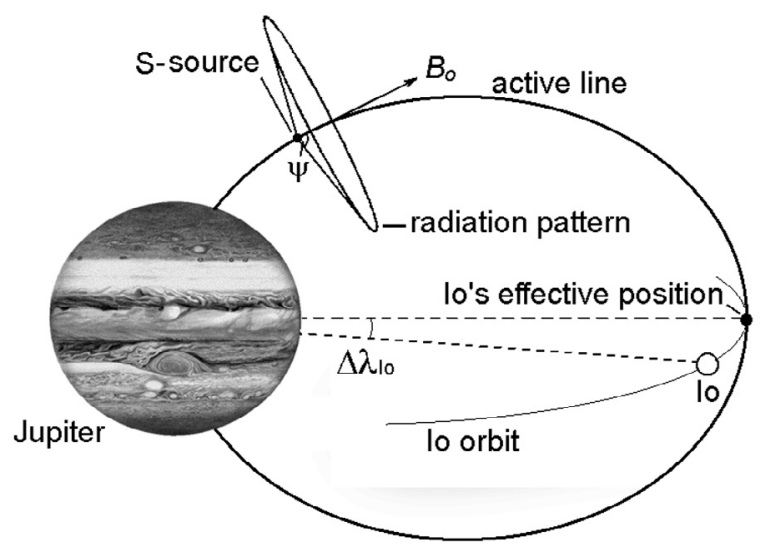

Fig. 1. The scheme of S-burst source localization and its radiation pattern according to the standard model (not to scale).

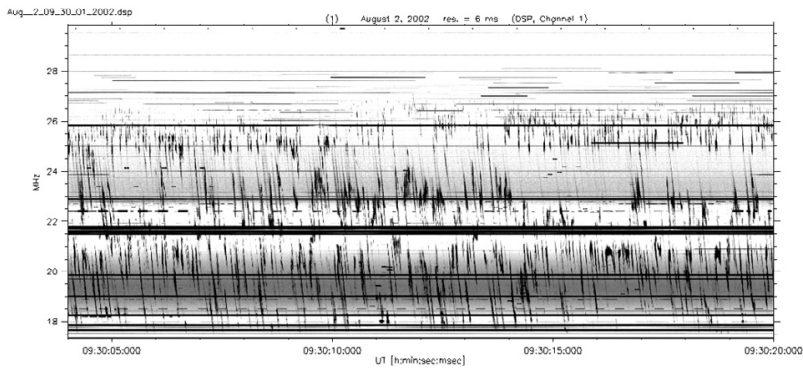

Fig. 2. The sample of S-bands at $f \approx 20,23$ and $25.5 \mathrm{MHz}$ in the dynamical spectrum of August 2, 2002, which was recorded with the UTR-2 radio telescope by an observation campaign within the frame of the INTAS project 03-51-5727.

interaction between a radio wave and a spiralling electron occurs when, in the frame where the electron's center of gyration is stationary, the cyclic frequency of the wave $(\omega)$ is equal to the gyrofrequency $\Omega_{\mathrm{e}} / \gamma$, where $\gamma=\sqrt{1-(v / c)^{2}}$ is the Lorentz factor ( $v$ and $c$ are the electron and light velocities, respectively). A resonant interaction occurs when the Doppler condition is satisfied,

$\omega-\frac{\Omega_{\mathrm{e}}}{\gamma}-k_{\|} v_{\|}=0$,

where $k_{\|}$and $v_{\|}$are the parallel (to the magnetic field) wave number of the radio wave and the parallel electron velocity, respectively.

As $v^{2}=v_{\|}^{2}+v_{\perp}^{2}$, the approximation $1 / \gamma \simeq 1-\frac{v_{\|}^{2}}{2 c^{2}}-\frac{v_{\perp}^{2}}{2 c^{2}}$ for $v \ll c$ in Eq. (1) gives the equation of resonant curve in the $v_{\|}-v_{\perp}$ plot,

$\frac{v_{\perp}^{2}}{c^{2}}+\left(\frac{v_{\|}}{c}-\frac{k_{\|} c}{\Omega_{\mathrm{e}}}\right)^{2}=\left(\frac{k_{\|} c}{\Omega_{\mathrm{e}}}\right)^{2}-\frac{2\left(\omega-\Omega_{\mathrm{e}}\right)}{\Omega_{\mathrm{e}}}$,

this is a semicircle centered on $v_{\perp} / c=0$ and $v_{\mathrm{o}} / c=k_{\|} c / \Omega_{\mathrm{e}}$. As $k_{\|}=k \cos \theta$ and $k=\omega n_{\mathrm{r}} / c$, we obtain $\frac{v_{0}}{c}=\frac{n_{\mathrm{r}} \omega}{\Omega_{\mathrm{e}}} \cos \theta$, where $n_{\mathrm{r}}$ is the refractive index. Unfortunately, the conditions in sources of S-bursts are unknown. However, it is generally accepted that $\omega \simeq \Omega_{\mathrm{e}}$ for DAM there (e.g., Zarka 1998). If there is some optimal resonance semicircle, and the refraction is moderate $(n \approx 1)$, then the radio emission is amplified most effectively in the hollow cone with the axis parallel to the local magnetic field and the half-angle of $\theta=\arccos \left(v_{\mathrm{o}} / c\right) \approx \frac{\pi}{2}-v_{\mathrm{o}} / c$ for semi-relativistic electrons.



Fig. 3. Modulation of S-emission flux by the radiation pattern swinging in the nodes of standing Alfvén wave. The maximal curves (not to scale) of the active magnetic line and the directions of connected radio beams are shown with half-period time interval (solid and dashed). Periodical orientation of radio beams to the Earth from several nodes forms the S-bands in dynamic spectrum at corresponding electron cyclotron frequencies (squares). Note the anti-correlation between radio beam directions or squares in adjacent S-bands.

The parallel electric field of the Alfvén wave accelerates electrons in the radio source. Hence, the electron distribution is shifted along the $v_{\|}$axis with $v_{\mathrm{o}} / c$. As a result, the variation of radio beam direction is $\Delta \theta \approx \Delta v_{\mathrm{o}} / c$, where $\Delta v_{\mathrm{o}}$ is the shift of the center of optimal resonant curve. It is found, experimentally, that the cone mantle thickness of $\mathrm{S}$-burst radiation pattern is $\Delta \vartheta \approx 1^{\circ}$, especially in Io-B radio storms (Ellis 1982; Ryabov 1994; Zarka 1998). Presumably, such a narrow radio beam can be a result of some compact detail of unknown electron pattern on the $v_{\|}-v_{\perp}$ plot, e.g., a narrow horseshoe feature or an electron beam (Fig. 3 in Hess et al. 2007b). Therefore, the radio beam could be turned away from the Earth if $\Delta v_{\mathrm{o}} / c \geq \Delta \theta \approx 1^{\circ} / 57.3^{\circ}=0.017 \mathrm{rad}$. This $\Delta v_{\mathrm{o}} / c$ estimation corresponds to the variation of electron energy of $\Delta E \geq 2 E \Delta v_{\mathrm{o}} / v_{\|} \approx 0.7 \mathrm{keV}$, where $E_{\|}=1.8 \mathrm{keV}$ and $V_{\|} / c=0.084$ are the mean parallel energy and corresponding parallel velocity of electrons emitting S-bursts at $f=20$ to $25 \mathrm{MHz}$ (Fig. 3d in Hess et al. 2007a). Sufficient electric potential jumps of 0.7 to $3 \mathrm{keV}$ are found experimentally from the analysis of the frequency drift rate of individual S-bursts (Fig. 6 in Hess et al. 2007a). These jumps are localized at quasi-stable altitudes, and they could be associated with nodes of the standing Alfvén wave.

Alternative models with strong S-burst refraction $\left(n_{\mathrm{r}} \ll 1\right.$ in the radio source; Boev et al. 1993; Zaitsev et al. 1986) predict that narrow radio beam is quasi-perpendicular to the local magnetic field. If the amplitude of magnetic deflection exceeds the cone mantle thickness of S-burst radiation pattern, the Alfvén wave can turn the radio beam away from the Earth as is shown in Fig. 3. It is possible with $\frac{180^{\circ}}{\pi} \Delta B / B \geq 1^{\circ}$, where $B \approx 0.7 \mathrm{mT}$ is the magnetic induction at $f \simeq f_{\text {ce }}=20 \mathrm{MHz}$, and $\Delta B \geq 0.012 \mathrm{mT}$ is the sufficient amplitude of the Alfvén wave. Unfortunately, the amplitude of magnetic pulsations in the regions of DAM sources is not measured yet.

Therefore, the cyclotron maser instability and alternative mechanisms assume the control of S-burst visibility by the standing Alfvén wave. The regions of easy observation of S-bursts coincide with the standing wave nodes. As the nodes are positioned quasi-periodically in space, corresponding S-trains must be quasi-periodical with frequency in dynamic spectrum. It is important that the parallel electric currents and transversal magnetic disturbances have opposite directions in adjacent nodes. Correspondingly, the radio beams must deviate in 
opposite directions, independent of emission mechanism. Such modulation in the standing wave model leads to the anticorrelation of radio fluxes in adjacent $\mathrm{S}$-trains or to the correlation with next (after adjacent) S-band (Fig. 3). This correlation pattern is a good test for the model.

To verify this test, we used the record of DAM spectral intensity at fixed frequencies in the centers of S-bands registered during 59.148 s with resolution of $6 \mathrm{~ms}$ at August 2, 2002, 9:30 UT (see part of these observations in Fig. 2).

The null hypothesis (no correlation) may be verified with the standard transformation of correlation coefficient $r_{\text {corr }}$ into the value $w=\frac{1}{2} \ln \frac{1+r_{\text {corr }}}{1-r_{\text {corr }}}$, which has the normal distribution (e.g., Bendat \& Piersol 1986). If no correlation exists between spectral intensity in two frequency channels, the average of $w$ distribution is zero, and its dispersion is $\sigma_{w}=1 / \sqrt{N-3}$, where $N=9983$ is the number of measurements in one channel. The probability $(\alpha)$ of deviation $w / \sigma_{w}>Z_{\alpha}$ is well known for the normal distribution: $\alpha=\int_{Z_{\alpha}}^{\infty} \frac{1}{2 \pi} \exp \left(-z^{2} / 2\right) \mathrm{d} z$. Hence, the confidence interval with $2 \alpha$-significance is $\frac{\eta_{2}-1}{1+\eta_{2}}<w<\frac{\eta_{1}-1}{1+\eta_{1}}$, where $\eta_{1,2}=\exp \left( \pm \frac{2 Z_{\alpha}}{\sqrt{N-3}}\right)$. In our case $\left(N \gg 1\right.$ and $\left.r_{\text {corr }} \ll 1\right)$ we have $w \simeq r_{\text {corr }}$ and $\eta_{1,2}=1 \pm \frac{2 Z_{\alpha}}{\sqrt{N}} \simeq 1$. Hence, the confidence interval of the null hypothesis can be approximated as $-\frac{Z_{\alpha}}{\sqrt{N}}<r_{\text {corr }}<\frac{Z_{\alpha}}{\sqrt{N}}$. Its borders $( \pm 0.038)$ are calculated with $\alpha=0.0005$ and $Z_{\alpha}=3.73$. Correspondingly, the null hypothesis predicts that $-0.038<r_{\text {corr }}<0.038$, with the probability of $1-2 \alpha=0.999$.

However, our experimental estimations of $r_{\text {corr }}$ show systematical deflections from the null prediction (Fig. 4). Moreover, there are significant spikes (arrowed) far out the confidence interval. Therefore, the null hypothesis is not valid, and some correlation pattern is present.

It is important that the found correlation details agree with the prediction of the standing wave model. There is a slight, but significant anti-correlation between adjacent $\mathrm{S}$-bands up to $r_{\text {corr }}=-0.09 \pm 0.01$ at $f=20.5$ and $23.1 \mathrm{MHz}$ with the time shift of $\Delta t=0.172 \mathrm{~s}$; and $r_{\text {corr }}=-0.09 \pm 0.01$ at $f=23.1$ and $25.25 \mathrm{MHz}$ with $\Delta t=0.154 \mathrm{~s}$. However, there is a positive correlation up to $r_{\text {corr }}=+0.09 \pm 0.01$ between the extreme $S$-bands (20.5 and $25.25 \mathrm{MHz}$ ) with $\Delta t=0.326 \mathrm{~s}$.

The mentioned time shift $\Delta t$ of maximal correlation corresponds to the drift rate of $\Delta f / \Delta t=(25.25 \mathrm{MHz}-$ $20.5 \mathrm{MHz}) / 0.326 \mathrm{~s}=-14.6 \mathrm{MHz} / \mathrm{s}$. Obviously this is the drift rate of S-bursts that typically equals from -10 to $-30 \mathrm{MHz} / \mathrm{s}$ (Hess et al. 2007a). Apparently, there is radio flux modulation along space trajectories of S-sources.

Figure 5 demonstrates other examples of anti-correlation of fine structure in adjacent S-bands at August 9, 2002. Hence, the model prediction is formally confirmed.

Apparently, the low level of anti-correlation in Figs. 4 and 5 is a result of the following interference factors.

(a) Moving disturbances from progressive magnetohydrodynamic waves of ultra-low frequencies (ULF) can complicate the situation. Such ULF modulating waves are found in DAM dynamic spectra as a moving pattern on the background of S-burst train. Their frequency drift corresponds to the wave motion to Jupiter with Alfvén velocity (Arkhypov \& Rucker 2007b).

(b) The excitation of several harmonics simultaneously in the Jovian ionospheric resonator can superimpose, or smooth over, nodes. The co-existence of different harmonics in this region is predicted theoretically ( $\mathrm{Su}$ et al. 2006) and

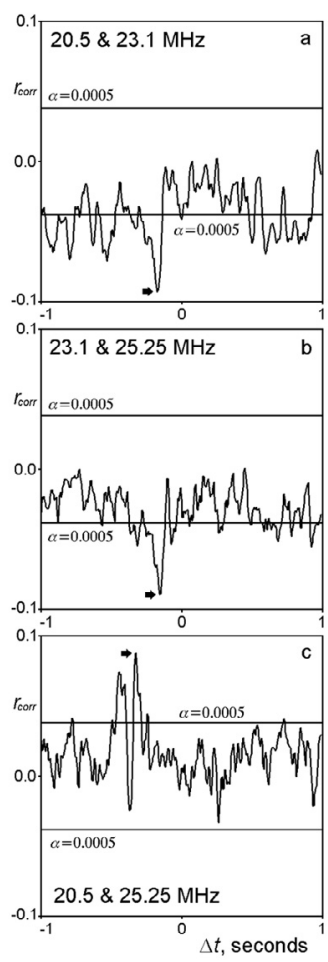

Fig. 4. Correlation coefficient $\left(r_{\text {corr }}\right)$ between DAM spectral intensity in different S-bands (marked frequencies) which are shown in Fig. 2. The coefficient has deep drops (arrowed) at approximately same time shifts $\Delta t$ between radio fluxes in adjacent $S$-bands (a), b)). However, there is a peak of positive correlation with the flux in the next nearest $S$ band (c)). The horizontal lines are the borders of the confidence interval for the null hypothesis (no correlation) with the probability of leakage marked.

confirmed experimentally as regular peaks in the Fourier spectrum of ULF variations of S-burst emission (Arkhypov \& Rucker 2006).

Although the found anti-correlation in adjacent S-bands is rather low, this effect does certainly exist. It should be described as an earlier unknown and an important argument for the connection between different S-bands.

\section{Macrostructure}

The average frequencies of S-bands show variations with the time scale of a few minutes (Boudjada et al. 1995). From the standing wave model it follows that the drift in frequency of the S-band reflects the spatial motion of the node (see Sect. 2). As the node is part of a standing wave, its motion must be in coordination with displacements of other nodes. Hence, the frequency variations of S-bands must be correlated.

Moreover, the simple relation between frequency displacements of two S-bands can be predicted. We consider the short (i.e., quasi-homogenous) part of an Alfvénic resonator with $X$ length that is equal to $N$ Alfvén semi-wavelengths along the active magnetic line, above the low border of the Jovian ionosphere. Then, the distance between adjacent nodes is $d=X / N$; and the coordinate of the node is $x=(n+1 / 2) X / N$, where $n=0$, 1,2 is the node's number. The $1 / 2$-addition takes into account the boundary condition of zero electrical current at the low border of ionosphere, which is between the wave nodes. If the resonator length is variable, their variations are $\delta d=\delta X / N$ and 

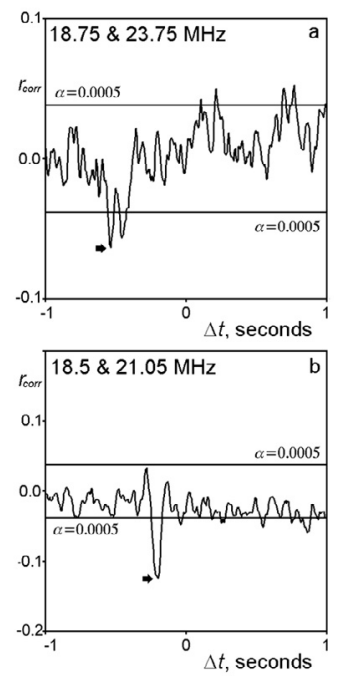

Fig. 5. Other examples of anti-correlation (arrowed) of microstructure in adjacent S-bands at August 9, 2002, 11:00 UT (a)) and 11:15 UT (b)). The dynamic spectrum was recorded with the UTR-2 radio telescope with an observation campaign within the frame of the INTAS project 03-51-5727. Marked like Fig. 4.

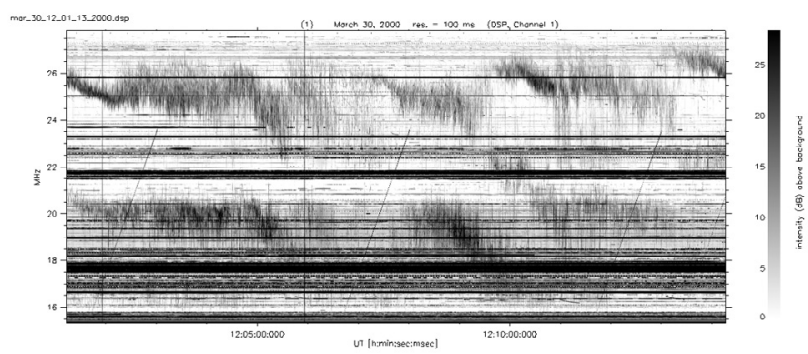

Fig. 6. Macrostructure of S-bands in dynamic spectrum of March 30, 2000 (UTR-2; INTAS 03-51-5727).

$\delta x=(n+1 / 2) \delta X / N$. Accordingly, the variation ratio of these parameters is $\delta d / \delta x=1 /(n+1 / 2)$. In case of $\delta x \ll R_{\mathrm{J}}$ and $\delta d \ll R_{\mathrm{J}}$ (where $R_{\mathrm{J}}$ is the Jovian radius), the linear approximation could be used to change over to the frequency scale: $\delta f=-\left|\mathrm{d} f_{\text {ce }} / \mathrm{d} x\right| \delta x$ and $\delta(\Delta f)=\left|\mathrm{d} f_{\text {ce }} / \mathrm{d} x\right| \delta d$. Therefore, the variation of frequency interval between adjacent S-bands $\delta(\Delta f)$ must be connected with the variation of S-band frequency $(\delta f)$ :

$\delta(\Delta f)=K \delta f$

where $K=-1 /(n+1 / 2)$.

To verify this prediction, we used the dynamic spectrum of DAM spectral intensity $(F)$ with two S-bands, which was recorded with the resolution of $0.1 \mathrm{~s}$ March 30, 2000, 12:01-14 UT (Fig. 6). We calculated the average gradient $\partial F / \partial f$ in the band of $0.56 \mathrm{MHz}$ (20 pixels) around each pixel of the spectrum. At the same time,we excluded the intensity bursts at fixed frequencies as a terrestrial interference. As a result, the scheme of maximal S-burst intensity in S-bands is constructed (Fig. 7).

The correlation coefficient between frequencies of different S-bands in Fig. $7 \mathrm{a}$ is $+0.789 \pm 0.012$. Hence, the first prediction on correlation between frequency shifts of different S-bands is confirmed.

The second prediction Eq. (3) is verified with the $\Delta f-$ $f$ diagram (Fig. 7b), which is constructed from Fig. 7a data. The correlation coefficient between $\Delta f$ and $f$ is $-0.43 \pm 0.04$.
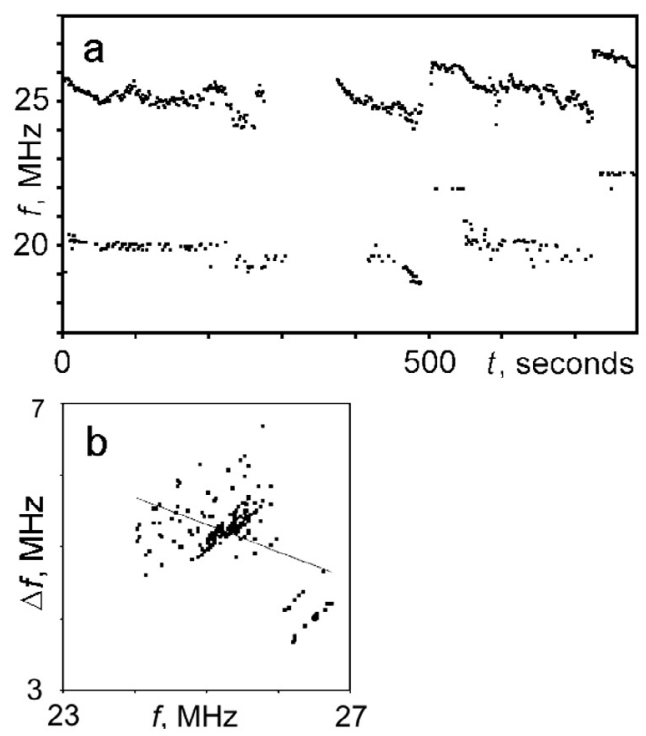

Fig. 7. The scheme of maximal intensity in S-bands from Fig. 6 (a)) and the corresponding relation between the S-band distance $(\Delta f)$ and the S-band frequency $(f)(\mathbf{b}))$. The line is the regression found with the method of least-squares.

The regression is found with the method of least-squares for 185 estimations:

$\Delta f=A+K f$

where $A=14.8 \pm 0.8 \mathrm{MHz}$ and $K=-0.38 \pm 0.03$. This estimation of $K$-parameter coincides with the expectation at $n=2$, according to Eq. (3): $K=-1 /(n+1 / 2)=-0.40$. Obviously, the differentiation transforms the regression Eq. (4) into relation Eq. (3). Hence, the second prediction on anti-correlation and the relation between variations of $\Delta f$ and $f$ of S-bands is confirmed as well.

The variability of S-band frequency could be interpreted in terms of a variable length of ionospheric Alfvén resonator near Jupiter. As it is near the Earth, this resonator could operate in a vertical direction with shear Alfvén waves, which are trapped between the ionosphere bottom and the peak of the Alfvén phase speed (Su et al. 2006).

The altitude of Alfvén speed maximum is controlled by the plasma mass density in the resonator. However, the parallel electric fields deplete the plasma density in flux tubes by about an order of magnitude. Such field-aligned cavities are found in the Io torus (Russel et al. 2005; Chust et al. 2005) and predicted theoretically in the connected regions of DAM sources ( $\mathrm{Su}$ et al. 2003). It is generally accepted to associate plasma cavities with DAM and its analogs on other planets (e.g., Zarka 1998). As S-bursts are stimulated by Io satellite (Zarka 1998), the S-source follows behind Io through various cavities in the low magnetosphere. Hence, the longitudinal variations in plasma density could modulate in time the length of the radio-active part of the Alfvén resonator, without physical variation of the length of the Alfven resonator. We receive S-bursts from various resonators one after another with different longitudes and lengths.

We calculate Alfvén speed $\left(V_{\mathrm{A}}\right)$ at various altitudes along the active magnetic field line to find its maximal value numerically:

$V_{\mathrm{A}}=\frac{1}{\sqrt{v_{\mathrm{a}}^{-2}+c^{-2}}}$,

where $v_{\mathrm{a}}=B / \sqrt{4 \pi m_{\mathrm{i}} n_{\mathrm{i}}} ; m_{\mathrm{i}}$ and $n_{\mathrm{i}}$ are the mass and number density of ions (mainly protons near Jupiter); $B$ is the magnetic 


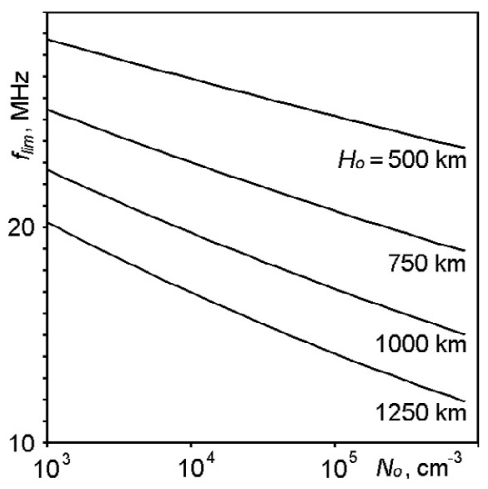

Fig. 8. The calculated $f_{\text {lim }}=f_{\text {ce }}$ at the altitude of maximal $V_{\mathrm{A}}$ with various peak electron density in the Jupiter's ionosphere $\left(N_{\mathrm{o}}\right)$ and the scale height $\left(H_{\mathrm{o}}\right)$. The date and time are identical to Figs. 6 and 7. The typical $\Delta \lambda_{I o}=39^{\circ}$ is accepted according to Arkhypov \& Rucker (2007a).

induction; $c$ is the light speed (e.g., Nicholson 1983). It could be accepted that $n_{\mathrm{i}}=N_{\mathrm{e}}$, where the electron number density $N_{\mathrm{e}}$ in the diffusion equilibrium model (Melrose 1967) is:

$N_{\mathrm{e}}=N_{\mathrm{o}} \exp \frac{-\left(Z-Z_{\mathrm{o}}\right)}{H_{\mathrm{o}}}$,

where $N_{\mathrm{o}}$ is the electron number density at height $Z_{\mathrm{o}}$, and $H_{\mathrm{o}}=$ $2 k_{\mathrm{b}} T /\left(m_{\mathrm{i}} g\right)$ is the scale height $\left(k_{\mathrm{b}}\right.$ is the Boltzmann constant; $T$ is the temperature). The geometric altitude in Eq. (6) is modified into corrected altitude $Z$, considering the spherical geometry and the influence of centrifugal force on co-rotating plasma (Melrose 1967):

$Z=R_{\mathrm{J}}\left(1-R_{\mathrm{J}} / r\right)-\left(\Omega R_{\mathrm{J}}\right)^{2}\left(r^{3} / R_{\mathrm{J}}^{3}-1\right) /(2 L g)$,

where $R_{\mathrm{J}}$ is the Jovian equatorial radius; $r$ is the planetocentric distance; $\Omega$ is the angular velocity of Jupiter's rotation; $L=6$ is the McIlwain parameter for the Io-related S-burst sources; and $g$ is the acceleration of gravity in the Jovian exobase.

The border of the resonator or $f_{\text {lim }}=f_{\text {ce }}$ at the altitude of maximal $V_{\mathrm{A}}$ is calculated with Eqs. (5)-(7) for various $N_{\mathrm{o}}$ and $H_{\mathrm{o}}$ with the VIP4 magnetic model (Connerney 1998) and a northern radio source. The result curves are shown in Fig. 8. It is follows from Fig. 8 that $f$ variations of S-bands $(2-3 \mathrm{MHz}$ in Fig. 7a) correspond to a variation of about a factor 10 in $N_{\mathrm{e}}$ or $\sim 20 \%$ in $H_{\mathrm{o}}$ or $T$. Such plasma caverns have been found in the Io plasma torus (Russell et al. 2005) and in the terrestrial polar magnetosphere (Zarka 1998).

Alfvénic oscillations in the radio source can modify the DAM radiation pattern (Fig. 3). After averaging the standing wave period, the conical sheet of radiation is thickening near the wave nodes. However, the radiation pattern is practically not modified at the wave maxima. As a result, we can see $\mathrm{S}$-bands in the dynamic spectrum. This S-band pattern evolves with the motions of the observer and the active magnetic line.

To model such evolution for a certain time interval, we tracked the active magnetic line numerically from the bottom of the Jovian ionosphere. We calculated the angle $\psi$ between the local magnetic field and the Earth direction at various points on the line. Ideally, we receive S-emission if $\psi_{\min }<\psi<\psi_{\max }$, where $\psi_{\min }$ and $\psi_{\max }$ are the borders of undisturbed S-radiation pattern. As the radiation pattern is thickening by the Alfvén standing wave, the condition of observability is $\psi_{\min }-\Delta \psi<\psi<$ $\psi_{\max }+\Delta \psi$. The harmonic (simplest) disturbance of active line is assumed. Hence, the Alfvén widening of the radiation pattern is suggested in the form of $\Delta \psi=A_{\psi}|\sin (2 \pi x / \lambda)|$, where $A_{\psi}$ is the

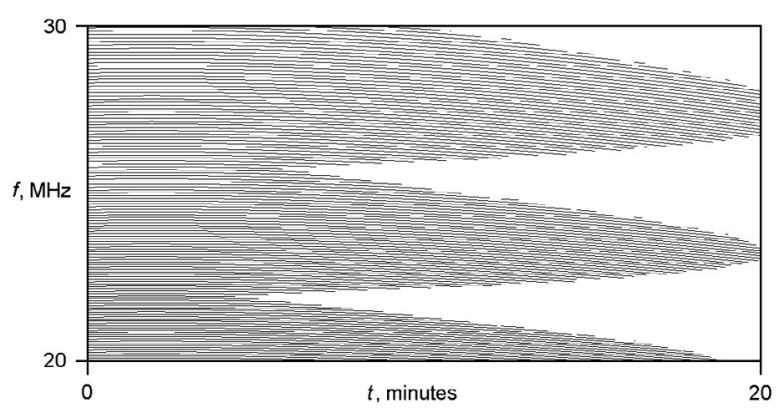

Fig. 9. The sample model of S-band evolution in the dynamic spectrum of March 30, 2000, 12:01 to 12:20 UT $\left(\psi_{\min }=72^{\circ} ; \psi_{\max }=73^{\circ} ; A_{\psi}=\right.$ $0.05 \mathrm{rad} ; \lambda=7000 \mathrm{~km}$ ). The area of S-emission (shaded) transforms from the broadband S-storm (left) to the S-bands (right).

amplitude of $\Delta \psi ; x$ is the distance from the ionosphere bottom along the active line; $\lambda$ is the Alfvén wavelength.

After calculations for different consecutive time moments with the VIP4 model (Connerney 1998), we find the sample distribution of S-emission in the dynamic spectrum (Fig. 9). In Fig. 9, broadband S-storm transforms into several S-bands. Such transformations were indeed described with real S-storm spectra (Riihimaa 1991).

\section{Conclusions}

There are theoretical and experimental arguments for a modulation of S-emission by standing Alfvén waves in the regions of Jovian decametric radio sources. One effect of such modulation is the phenomenon of S-bands in the dynamic spectra of DAM. We argue that S-bands are generated around nodes of a standing Alfvén wave. To test this hypothesis, we derive the following predictions for empirical verification.

1. The standing wave model predicts the anti-correlation between the S-band spectral intensity and the intensity in the adjacent band at short time scales $(<0.1 \mathrm{~s})$. We predict a positive correlation with the next band of S-bursts. This effect is found in two S-storms (August 2 and 9, 2002) as the significantly negative, or respectively positive, narrow impulse of the correlation between different S-bands at the time shift corresponding to the frequency drift rate of individual S-bursts.

2. At long time scales $(>0.1 \mathrm{~s})$, we predict the correlated frequency displacements of adjacent $\mathrm{S}$-bands as the spatial motion of the node system. We confirm such correlation $\left(r_{\text {corr }}=\right.$ $+0.789 \pm 0.012$ ) during the S-storm of March 30, 2000 .

3 . If the average frequency $(f)$ of the $S$-band increases, the frequency distance between adjacent S-bands $(\Delta f)$ decreases. This relation is a result of shortening the resonator part that is connected with the observed S-bursts. Such anti-correlation is confirmed ( $\left.r_{\text {corr }}=-0.43 \pm 0.04\right)$. Moreover, the regression between $f$ and $\Delta f$ practically coincides with the predicted relation for the second node counted from the planet.

4. An alfvénic oscillation in the radio source broadens the average radiation pattern near the wave nodes, but such modification does not exist at the standing wave maxima. The observer receives the broadband $\mathrm{S}$-storm in the main radio beam. Then, the beam turns away from the Earth, and the observer can register emission only from the nodes of the standing Alfvén wave as a system of S-bands. Such 
transformation of broadband S-storm into S-bands is well known (e.g. Riihimaa 1991).

As the predictions are confirmed, the standing wave modulation of Jovian S-emission appears valid.

Acknowledgements. We used the dynamical spectra of S-storms, which have been recorded by an observation compaign within the frame of the INTAS project 03-51-5727 (A. Lecacheux, H. O. Rucker, A. A. Konovalenko and Yu. Tokarev) with the UTR-2 radio telescope. We thank Dr. V. E. Shaposhnikov for fruitful discussions.

\section{References}

Arkhypov, O. V., \& Rucker, H. O. 2006, A\&A, 452, 347 Arkhypov, O. V., \& Rucker, H. O. 2007a, A\&A, 467, 353

Arkhypov, O. V., \& Rucker, H. O. 2007b, A\&A, 474, 1031

Boev, A. G., Luk'yanov, M. Yu., \& Tsvyk, N. A. 1993, Kinematika i Fizika Nebesnykh Tel. 1993, 9, 27

Bendat, J. S., \& Piersol, A. G. 1986, Random data, Analysis and measurement procedures (N.Y.: Wiley \& Sons), Ch. 4.8.1

Boudjada, M. Y., Rucker, H. O., Ladreiter, H. P., \& Ryabov, B. P. 1995, A\&A, 295, 782

Chust, T., Roux, A., Kurth, W. S., et al. 2005, Planet. and Space Sc., 53, 395

Connerney, J. E. P., Acuna, M. H., Ness, N. F., \& Satoh, T. 1998, J. Geophys. Res., 103, A6, 11929
Ellis, G. R. A. 1982, Aust. J. Phys., 35, 2, 165

Ergun, R. E., Su, Y.-J., Andersson, L., et al. 2006, J. Geophys. Res., 111, A6, A062212

Hess, S., Zarka, P., \& Mottez, F. 2007a, Planet. and Space Sci., 55, 89

Hess, S., Mottez, F., \& Zarka, P. 2007b, J. Geophys. Res., 112, A11, A11212

Leblanc, Y. 1981, J. Geophys. Res., 86, A10, 8546

Leblanc, Y., \& Genova, F. 1981, J. Geophys. Res., 86, A10, 8564

Litvinenko, G. V., Rucker, H. O., Vinogradov, V. V., Leitner, M., \& Shaposhnikov, V. E. 2004, A\&A, 426, 343

Melrose, D. B. 1967, Planet. Space Sci., 15, 2, 381

Nicholson, D. R. 1983, Introduction to plasma theory (N.Y.: Wiley \& Sons), 163

Riihimaa, J. J. 1991, Earth, Moon, and Planets, 53, 2, 157

Russell, C. T., Kivelson, M. G., \& Khurana, K. K. 2005, Planet. and Space Sci., 53, 937

Ryabov, B. P., Arkhipov, A. V., \& Shevchenko, V. A. 1985, Astron. Vestn., 19, 296

Ryabov, B. P. 1994, J. Geophys. Res., 99, E4, 8441

Ryabov, B. P., Zarka, P., Rucker, H. O., Ryabov, V. B., \& Boudjada, M. Y. 1997, Recurrent fine structures in Jovian S-burst emission, in Planetary Radio Emissions IV, ed. H. O. Rucker, S. J. Bauer, \& A. Lecacheux (Vienna: Austrian Acad. Sc. Press), 65

Su, Y.-J., Ergun, R. E., Bagenal, F., \& Delamere, P. A. 2003, J. Geophys. Res. 108, A2, 1094

Su, Y.-J., Jones, S. T., Ergun, R. E., et al. 2006, J. Geophys. Res. 111, A6, A06211

Zaitsev, V. V., Zlotnik, E. Y., \& Shaposhnikov, V. E. 1986, A\&A, 169, 345

Zarka, P. 1998, J. Geophys. Res., 103, E9, 20159 